\title{
A Priori and A Posteriori Error Estimates for a Crank Nicolson Type Scheme of an Elliptic Problem with Dynamical Boundary Conditions
}

\author{
Rola Ali Ahmad ${ }^{1}$, Toufic El Arwadi ${ }^{1}$, Houssam Chrayteh ${ }^{1} \&$ Jean-Marc Sac-Epée $^{2}$ \\ ${ }^{1}$ Beirut Arab University, Lebanon \\ ${ }^{2}$ Lorraine University, France \\ Correspondence: Toufic El Arwadi, Beirut Arab University, Lebanon. E-mail: t.elarwadi@bau.edu.lb
}

Received: December 22, 2015 Accepted: January 19, 2016 Online Published: March 9, 2016

doi:10.5539/jmr.v8n2p1 URL: http://dx.doi.org/10.5539/jmr.v8n2p1

\begin{abstract}
In this article we claim that we are going to give a priori and a posteriori error estimates for a Crank Nicolson type scheme. The problem is discretized by the finite elements in space. The main result of this paper consists in establishing two types of error indicators, the first one linked to the time discretization and the second one to the space discretization.
\end{abstract}

Keywords: A priori and a posteriori error estimates, Crank Nicolson type scheme, finite elements

\section{Introduction}

Let $\Omega$ be a bounded smooth sub domain of $\mathbb{R}^{\not}$ and $\gamma(x)=\left[\gamma_{i, j}\right]_{i, j=1}^{n}$ be a real positive definite matrix-valued function. Let $(0, T)$ denote a subinterval of $R$ where $T \in(0, \infty)$ is a fixed final time. Denote by $n(x)$ the unit outward normal vector at $x \in \Gamma$. We intend to work with the following problem,

$$
\left\{\begin{array}{l}
\operatorname{div}(\gamma \nabla u)=0, \text { in }(0, T) \times \Omega \\
\frac{\partial u}{\partial t}(t, x)+\gamma n(x) \cdot \nabla u(t, x)=0, \text { on }(0, T) \times \Gamma \\
u(0, x)=u_{0}(x), \text { on } \Gamma
\end{array}\right.
$$

where $\Gamma$ is the boundary, $u$ is the unknown and $u_{0}$ is the initial condition at time $t=0$.

The solution of the above problem can be represented on the boundary by the Dirichlet-to-Neumann semigroup (Vrabie, 2003) defined as

$$
(S(t) f)(x)=\left.u(t, x)\right|_{\Gamma}
$$

In (Cherif, Arwadi, Emmamirad \& Sac Epee, 2014), the authors showed that the Lax semigroup is the Dirichlet-toNeumann semigroup in the particular case where $\Omega=B(0,1)$ is the unit ball of $\mathbb{R}^{\not t}$ and $\gamma(x)$ is the identity matrix. P. Lax showed in his book (Lax, 2002) that the DtN semigroup has an explicit representation. This was a motivation for the authors in (Cherif, Arwadi, Emmamirad \& Sac Epee, 2014) and (Emmamirad \& Shariftabbar, 2013) to introduce semi discrete implicit and explicit Euler's schemes to approximate the DtN semigroup numerically. They also showed the convergence of these schemes using the Chernoff's product formula.

For more than twenty years, an impressive amount of work has been accomplished concerning a posteriori analysis and mesh adaptivity for the finite element discretization of the elliptic problems. Their main results were to exhibit local error indicators which can be computed explicitly as a function of the discrete solution and the data.

In (Arwadi, Dib \& Sayah, 2015), they studied the time dependent linear elliptic problem, and established optimal a priori and a posteriori error estimates using the backward Euler's scheme in time and finite elements in space.

The Crank Nicolson scheme is one of the most popular time-stepping method; however optimal a priori and a posteriori error estimates for elliptic equations have not yet been derived. The aim of this work is to provide optimal a priori and a posteriori estimates and some numerical investigations.

The term "a posteriori error estimator" was first used by Ostrowski (Ostrowski, 1940). It is the quantity which bounds or approximates the error, i.e. an upper bound of the error between an exact solution and a numerical one.

The error estimator is obtained as a sum of local indicators expressed on each element of the mesh (Mishra, 2012). We have two types of computable error indicators, the first being linked to the time discretization and the second to the space discretization.

We say that the a posteriori error estimates are optimal if we are able to bound each one of this indicators by the local 
error of the solution around the corresponding element. In this work, we propose a low cost discretization relying on the Crank Nicolson's scheme in time combined with the finite elements in space, and then prove a priori and a posteriori error estimates for the discrete problem.

The outline of the paper is as follows. In section 2, we give some notations that will be used in the sequel. Section 3 is devoted to study the discrete problem and the uniqueness of its solution. In section 4, we study the a priori errors and derive optimal estimates. Section 5 is devoted to study the a posteriori errors where two types of error indicators are established.

\section{Notations}

In this section we will introduce some notations that will be used in the sequel.

- $h$ the maximal diameter of the elements of all $\tau_{n h}$

- $h_{n}$ the maximal diameter of the elements of $\tau_{n h}$ for each $\mathrm{n}$

- $h_{\kappa}$ the diameter of $\kappa$

- $h_{e}$ the diameter of the edge $\mathrm{e}$

- $\Delta_{\kappa}$ the union of elements of $\tau_{n h}$ that intersect $\kappa$

- $\Delta_{e}$ the union of elements of $\tau_{n h}$ that intersect the edge e

- $\epsilon_{\kappa}$ the set of edges of $\kappa$ that are not on $\Gamma$

- $\epsilon_{\kappa}^{m}$ the set of edges of $\kappa$ that are on $\Gamma$

- $[.]_{e}$ the jump through e for each edge e in $\epsilon_{\kappa}$

- $\psi_{\kappa}$ the bubble function which is equal to the product of the three barycentric coordinates associated with the vertices of $\kappa$

- $L_{e}$ the lifting operator defined on polynomials on e vanishing on $\partial e$

- $X_{n h}$ the finite dimensional space of functions such that their restrictions to any element $\kappa$ of $\tau_{n h}$ belong to a space of polynomials of degree one. In other words,

$$
X_{n h}=\left\{v_{n}^{h} \in C^{0}(\bar{\Omega}),\left.v_{n}^{h}\right|_{\kappa} \text { isaffine } \forall \kappa \in T_{n h}\right\}
$$

- $I_{h}$ the approximation operator in $L\left(H^{2}(\Omega) ; X_{n h}\right)$ such that for $m=0,1$,

$$
\forall v \in H^{2}(\Omega),\left|I_{h}(v)-v\right|_{m, \Omega} \leq C h^{2-m}|v|_{2, \Omega}
$$

- We introduce the Sobolev spaces:

$$
H^{m}(\Omega)=\left\{v \in L^{2}(\Omega), \partial^{\alpha} v \in L^{2}(\Omega), \forall|\alpha| \leq m\right\},
$$

equipped with the following semi-norm and norm:

$$
|v|_{m, \Omega}=\left\{\sum_{|\alpha|=m} \int_{\Omega}\left|\partial^{\alpha} v(x)\right|^{2} d x\right\}^{\frac{1}{2}}
$$

and

$$
\|v\|_{m, \Omega}=\left\{\sum_{k \leq m}|v|_{k, \Omega}^{2}\right\}^{\frac{1}{2}}
$$




\section{The Discrete Problem}

Assume that $\Omega$ is a polyhedron and $\gamma$ denotes a positive smooth bounded function. We introduce a partition of the interval $[0, T]$ into sub intervals $\left[t_{n-1}, t_{n}\right], 1 \leq n \leq N$, such that $0=t_{0} \leq t_{1} \leq \ldots \leq t_{N}=T$. Denote by $\tau_{n}$ the length of $\left[t_{n-1}, t_{n}\right]$, by $|\tau|$ the maximum of the $\tau_{n}$, by $\tau$ the $\mathrm{N}$-tuple $\left(\tau_{1}, \ldots, \tau_{N}\right)$, and by $\sigma_{\tau}$ the regularity parameter

$$
\sigma_{\tau}=\max _{2 \leq n \leq N} \frac{\tau_{n}}{\tau_{n-1}} .
$$

Theorem 1 If $u(t) \in H^{2}(\Omega)$, then Problem (1) is equivalent to the variational problem,

$$
\left\{\begin{array}{l}
\text { Find } u(t) \in H^{1}(\Omega), \text { such that } \\
u(0, x)=u_{0}(x), \text { on } \Gamma \\
\int_{\Omega} \gamma \nabla u \nabla v d x+\int_{\Gamma} \frac{\partial u}{\partial t}(t, s) v(t, s) d s=0, \forall v(t) \in H^{1}(\Omega)
\end{array}\right.
$$

Proof. Let $u(t)$ be a solution of problem (1). Multiplying the first equation of problem (1) by $v(t) \in H^{1}(\Omega)$, integrating over $\Omega$, applying Green's formula and using the second equation of problem (1), we obtain that $u$ is also a solution of problem (2). Conversely, if $u$ is a solution of problem (2), we take $v(t) \in D(\Omega)$ to get the first line of problem (1). Then multiplying the first equation of problem (1) by $v(t) \in H^{1}(\Omega)$, integrating over $\Omega$, using the Green's formula and comparing with problem (2), we get the second line of problem (1).

Proposition 1 The solution of Problem (2) satisfies the following bound:

$$
\|u\|_{L^{\infty}\left(0, T, L^{2}(\Gamma)\right)}^{2} \leq\left\|u_{0}\right\|_{L^{2}(\Gamma)}
$$

Now, the full discrete problem associated to the variational problem (2) is:

$$
\left\{\begin{array}{l}
\text { Given } u_{h}^{n} \in X_{n h}, \\
\forall v_{h}(t) \in X_{n h}, u_{h}^{n}(t) \text { is the solution of } \\
\int_{\Omega} \gamma \nabla u_{h}^{n+1}(x) \nabla v_{h}(t, x) d x+2 \int_{\Gamma} \frac{u_{h}^{n+1}-u_{h}^{n}}{\tau_{n}}(x) v_{h}(t, x) d x-\int_{\Omega} \gamma \nabla u_{h}^{n} \nabla v_{h} d x=0
\end{array}\right.
$$

Theorem 2 The problem (3) admits a unique solution in $X_{n h}$.

Proof. We introduce the bilinear form,

$$
a\left(u_{h}^{n+1}, v_{h}\right)=\int_{\Omega} \tau_{n} \gamma \nabla u_{h}^{n+1} \nabla v_{h} d x+2 \int_{\Gamma} u_{h}^{n+1} v_{h} d \sigma
$$

and the linear form

$$
L\left(v_{h}\right)=\int_{\Omega} \tau_{n} \gamma \nabla u_{h}^{n} \nabla v_{h} d x+2 \int_{\Gamma} u_{h}^{n} v_{h} d \sigma
$$

Then the previous problem can be written as

$$
\forall v_{h} \in X_{n h}, a\left(u_{h}^{n+1}, v_{h}\right)=L\left(v_{h}\right)
$$

It is obvious that $a$ is bilinear and continuous in $X_{n+1, h} \times X_{n+1, h}$, and that $L$ is linear and continuous in $X_{n h}$ and then, the Lax-Milgram theorem states the existence and the uniqueness of the solution. See (Arwadi, Dib \& Sayah, 2015).

\section{A Priori Error Estimate}

To get an a priori error estimate, we need the following Gronwall's lemma.

Lemma 1 Gronwall's lemma:

Let $\left(a_{n}\right)_{n} \geq 0,\left(b_{n}\right)_{n} \geq 0$ and $\left(c_{n}\right)_{n} \geq 0$ be three real positive sequences such that $\left(c_{n}\right)_{n} \geq 0$ is an increasing sequence. Suppose that

$$
a_{0}+b_{0} \leq c_{0}
$$

there exists $\lambda>0$ such that:

$$
\forall n \geq 0, a_{n}+b_{n} \leq c_{n}+\lambda \sum_{m=0}^{n-1} a_{m}
$$


then we have

$$
\forall n \geq 0, a_{n}+b_{n} \leq c_{n} e^{n \lambda}
$$

Theorem 3 If $u \in L^{\infty}$ we have,

$$
\left\|u\left(t_{m+1}\right)-u_{h}^{m+1}\right\|_{0, \Gamma}^{2}+k\left|C_{\gamma}\right| \sum_{n=0}^{m}\left|u\left(t_{n+1}\right)-u_{h}^{n+1}\right|_{1, \Omega}^{2} \leq c\left(h^{2}+k^{2}\right)
$$

where $c$ is a constant independent of $h$ and $k$.

Proof. Denote by $k$ the time step, $h$ the parameter of the mesh and $X_{h}$ the discrete space. Suppose that $\tau_{n}$ and $\tau_{n h}$ are constants during time iterations. Consider the equation,

$$
\int_{\Omega} \nabla u(t, x) \nabla v(t, x) d x+2 \int_{\Gamma} \frac{\partial u}{\partial t}(t, s) v(t, s) d s=0, \forall v(t) \in H^{1}(\Omega)
$$

For $t \in\left(t_{n}, t_{n+1}\right)$ take $v=v_{h}^{n+1}$, integrate in time

$$
\int_{t_{n}}^{t_{n+1}} \int_{\Omega} \nabla u(t, x) \nabla v_{h}^{n+1}(t, x) d x+2 \int_{t_{n}}^{t_{n+1}} \int_{\Gamma} \frac{\partial u}{\partial t}(t, s) v_{h}^{n+1}(t, s) d s=0
$$

The discrete variation formulation for the Crank Nicolson scheme taken in the time step $n+1$, is

$$
\int_{\Omega} \gamma \nabla u_{h}^{n+1} \nabla v_{h}^{n+1} d x+2 \int_{\Gamma} \frac{u_{h}^{n+1}-u_{h}^{n}}{\tau_{n}} v_{h}^{n+1} d \sigma-\int_{\Omega} \gamma \nabla u_{h}^{n} \nabla v_{h}^{n+1} d x=0
$$

Integrating in time between $t_{n}$ and $t_{n+1}$ we get,

$$
\int_{t_{n}}^{t_{n+1}} \int_{\Omega} \gamma \nabla u_{h}^{n+1} \nabla v_{h}^{n+1} d x+2 \int_{t_{n}}^{t_{n+1}} \int_{\Gamma} \frac{u_{h}^{n+1}-u_{h}^{n}}{\tau_{n}} v_{h}^{n+1} d \sigma-\int_{t_{n}}^{t_{n+1}} \int_{\Omega} \gamma \nabla u_{h}^{n} \nabla v_{h}^{n+1} d x=0
$$

Taking the difference between (4) and (5) we get,

$$
\int_{t_{n}}^{t_{n+1}} \int_{\Omega} \gamma \nabla\left(u-u_{h}^{n+1}+u_{h}^{n}\right) \nabla v_{h}^{n+1} d x+2 \int_{\Gamma}\left[\left(u\left(t_{n+1}\right)-u\left(t_{n}\right)\right)-\left(u_{h}^{n+1}-u_{h}^{n}\right)\right] v_{h}^{n+1} d \sigma=0
$$

Now inserting $\pm \nabla\left(I_{h}\left(u\left(t_{n+1}\right)\right)\right), \nabla\left(I_{h}\left(u\left(t_{n}\right)\right)\right), \nabla\left(u\left(t_{n+1}\right)\right)$ and $\nabla\left(u\left(t_{n}\right)\right)$ into the first term, and $\pm I_{h}\left(u\left(t_{n+1}\right)\right)$ and $I_{h}\left(u\left(t_{n}\right)\right)$ into the second term, we obtain

$$
\begin{aligned}
& -\int_{t_{n}}^{t_{n+1}} \int_{\Omega} \gamma \nabla\left(u\left(t_{n+1}\right)-u\left(t_{n}\right)\right) \nabla v_{h}^{n+1} d x d t-\int_{t_{n}}^{t_{n+1}} \int_{\Omega} \gamma \nabla\left(I_{h}\left(u\left(t_{n+1}\right)\right)-u\left(t_{n+1}\right)\right) \nabla v_{h}^{n+1} d x d t \\
& +\int_{t_{n}}^{t_{n+1}} \int_{\Omega} \gamma \nabla\left(I_{h}\left(u\left(t_{n+1}\right)\right)-u_{h}^{n+1}\right) \nabla v_{h}^{n+1} d x d t-\int_{t_{n}}^{t_{n+1}} \int_{\Omega} \gamma \nabla\left(I_{h}\left(u\left(t_{n}\right)\right)-u_{h}^{n}\right) \nabla v_{h}^{n+1} d x d t \\
& \quad+\int_{t_{n}}^{t_{n+1}} \int_{\Omega} \gamma \nabla\left(I_{h}\left(u\left(t_{n}\right)\right)-u\left(t_{n}\right)\right) \nabla v_{h}^{n+1} d x d t+\int_{t_{n}}^{t_{n+1}} \int_{\Omega} \gamma \nabla\left(u\left(t_{n}\right)\right) \nabla v_{h}^{n+1} d x d t \\
& +2 \int_{\Gamma}\left(a_{n+1}-a_{n}\right)(s) v_{h}^{n+1} d s-2 \int_{\Gamma}\left(I_{h}\left(u\left(t_{n+1}\right)\right)-u\left(t_{n+1}\right)\right)-\left(I_{h}\left(u\left(t_{n}\right)\right)-u\left(t_{n}\right)\right) v_{h}^{n+1} d s=0
\end{aligned}
$$

where $a_{n+1}=I_{h}\left(u\left(t_{n+1}\right)\right)-u_{h}^{n+1}$ and $a_{n}=I_{h}\left(u\left(t_{n}\right)\right)-u_{h}^{n}$.

Now we will bound the third and fourth terms of the previous equation. Choosing $v_{h}^{n+1}=a_{n+1}$

$$
\begin{aligned}
\int_{t_{n}}^{t_{n+1}} \int_{\Omega} \gamma \nabla\left(I_{h}\left(u\left(t_{n+1}\right)\right)-u_{h}^{n+1}\right) \nabla v_{h}^{n+1} d x d t & =\int_{t_{n}}^{t_{n+1}} \int_{\Omega} \gamma \nabla a_{n+1} \nabla v_{h}^{n+1} d x d t \\
& =\int_{t_{n}}^{t_{n+1}} \int_{\Omega} \gamma \nabla a_{n+1}^{2} d x d t \\
& \leq k\left|C_{\gamma}\right|\left|a_{n+1}\right|_{1, \Omega}^{2}
\end{aligned}
$$




$$
\begin{aligned}
\int_{t_{n}}^{t_{n+1}} \int_{\Omega} \gamma \nabla\left(I_{h}\left(u\left(t_{n}\right)\right)-u_{h}^{n}\right) \nabla v_{h}^{n+1} d x d t & =\int_{t_{n}}^{t_{n+1}} \int_{\Omega} \gamma \nabla a_{n} \nabla a_{n+1} d x d t \\
& \leq k\left|C_{\gamma}\right|\left|a_{n}\right|_{1, \Omega}\left|a_{n+1}\right|_{1, \Omega}
\end{aligned}
$$

we obtain

$2 \int_{\Gamma}\left(a_{n+1}-a_{n}\right)(s) v_{h}^{n+1} d s+k\left|C_{\gamma}\right|\left|a_{n+1}\right|_{1, \Omega}^{2}-k\left|C_{\gamma}\right|\left|a_{n}\right|_{1, \Omega}\left|a_{n+1}\right|_{1, \Omega}$

$$
\begin{aligned}
& =2 \int_{\Gamma}\left(I_{h}\left(u\left(t_{n+1}\right)\right)-u\left(t_{n+1}\right)\right)-\left(I_{h}\left(u\left(t_{n}\right)\right)-u\left(t_{n}\right)\right) v_{h}^{n+1} d s \\
& +\int_{t_{n}}^{t_{n+1}} \int_{\Omega} \gamma \nabla\left(u\left(t_{n+1}\right)-u(t)\right) \nabla v_{h}^{n+1} d x d t \\
& +\int_{t_{n}}^{t_{n+1}} \int_{\Omega} \gamma \nabla\left(I_{h}\left(u\left(t_{n+1}\right)\right)-u\left(t_{n+1}\right)\right) \nabla v_{h}^{n+1} d x d t-\int_{t_{n}}^{t_{n+1}} \int_{\Omega} \gamma \nabla\left(I_{h}\left(u\left(t_{n}\right)\right)-u\left(t_{n}\right)\right) \nabla v_{h}^{n+1} d x d t \\
& -\int_{t_{n}}^{t_{n+1}} \int_{\Omega} \gamma \nabla\left(u\left(t_{n}\right)\right) \nabla v_{h}^{n+1} d x d t
\end{aligned}
$$

We denote by $T_{1}$ the first term of the left hand side, $T_{2}$ and $T_{3}$ the first and second terms of the right hand side, $T_{4}$ the third and fourth terms, and $T_{5}$ the last term of the equation.

The term $T_{1}$ can be expressed as

$$
\begin{aligned}
T_{1}=2 \int_{\Gamma}\left(a_{n+1}-a_{n}\right)(s) v_{h}^{n+1} d s & =2 \int_{\Gamma}\left(a_{n+1}^{2}-a_{n} a_{n+1}\right) d s \\
& =\int_{\Gamma} a_{n+1}^{2} d s-\int_{\Gamma} a_{n}^{2} d s+\int_{\Gamma}\left(a_{n+1}-a_{n}\right)^{2} d s
\end{aligned}
$$

The term $T_{2}$ can be bounded as

$$
\begin{aligned}
T_{2} & =2 \int_{\Gamma}\left(I_{h}\left(u\left(t_{n+1}\right)\right)-u\left(t_{n+1}\right)\right)-\left(I_{h}\left(u\left(t_{n}\right)\right)-u\left(t_{n}\right)\right) v_{h}^{n+1} d s \\
& =2 \int_{\Gamma}\left(g\left(t_{n+1}\right)-g\left(t_{n}\right)\right) a_{n+1} d s \\
& =2 \int_{t_{n}}^{t_{n+1}} \int_{\Omega} g^{\prime}(\tau, s) a_{n+1} d s d \tau \\
& \leq 2 \int_{t_{n}}^{t_{n+1}}\left\|g^{\prime}(\tau)\right\|_{0, \Gamma}\left\|a_{n+1}\right\|_{0, \Gamma} d \tau
\end{aligned}
$$

But

$$
\begin{aligned}
\left\|g^{\prime}(\tau)\right\|_{0, \Gamma} & \leq c\left\|g^{\prime}(\tau)\right\|_{1, \Omega} \\
& \leq \tilde{c} h\left\|u^{\prime}(\tau)\right\|_{2, \Omega} \\
& \leq c_{1} h\left\|u^{\prime}(\tau)\right\|_{L^{\infty}\left(0, T, H^{2}(\Omega)\right)}
\end{aligned}
$$

then,

$$
T_{2} \leq c_{1} h k\left\|u^{\prime}(\tau)\right\|_{L^{\infty}\left(0, T, H^{2}(\Omega)\right)}\left\|a_{n+1}\right\|_{0, \Gamma}
$$

Using the inequality $a b \leq \frac{1}{2 \epsilon_{1}} a^{2}+\frac{\epsilon_{1}}{2} b^{2}$,

with $a=c_{1} h \sqrt{k}\left\|u^{\prime}\right\|_{L^{\infty}}$ and $b=\sqrt{k}\left\|a_{n+1}\right\|_{0, \Gamma}$, we get

$$
T_{2} \leq \frac{1}{2 \epsilon_{1}} c_{1}^{2} h^{2} k\left\|u^{\prime}(\tau)\right\|_{L^{\infty}\left(0, T, H^{2}(\Omega)\right)}^{2}+\frac{\epsilon_{1}}{2} k\left\|a_{n+1}\right\|_{0, \Gamma}^{2}
$$


The term $T_{3}$ can be bounded as

$$
\begin{aligned}
T_{3} & =\int_{t_{n}}^{t_{n+1}} \int_{\Omega} \gamma \nabla\left(u\left(t_{n+1}\right)-u(t)\right) \nabla v_{h}^{n+1} d x d t \\
& =\int_{t_{n}}^{t_{n+1}} \int_{t}^{t_{n+1}} \int_{\Omega} \gamma \nabla\left(u^{\prime}(\tau, x)\right) \nabla a_{n+1} d x d \tau d t \\
& \leq \int_{t_{n}}^{t_{n+1}} \int_{t}^{t_{n+1}} \gamma\left\|u^{\prime}(\tau)\right\|_{1, \Omega}\left\|a_{n+1}\right\|_{1, \Omega} d \tau d t \\
& \leq k^{2}\left|C_{\gamma}\right|\left\|u^{\prime}\right\|_{L^{\infty}\left(0, T, H^{1}(\Omega)\right)}\left|a_{n+1}\right|_{1, \Omega}
\end{aligned}
$$

Using the inequality $a b \leq \frac{1}{2 \epsilon_{2}} a^{2}+\frac{\epsilon_{2}}{2} b^{2}$,

with $a=k^{\frac{3}{2}} C_{\gamma}\left\|u^{\prime}\right\|_{L^{\infty}}$ and $b=\sqrt{k}\left\|a_{n+1}\right\|_{1, \Omega}$, we get

$$
T_{3} \leq \frac{1}{2 \epsilon_{2}} k^{3} C_{\gamma}^{2}\left\|u^{\prime}\right\|_{L^{\infty}\left(0, T, H^{1}(\Omega)\right)}^{2}+\frac{\epsilon_{2}}{2} k\left|a_{n+1}\right|_{1, \Omega}^{2}
$$

Now the term $T_{4}$ can be bounded as

$$
\begin{aligned}
T_{4} & =\int_{t_{n}}^{t_{n+1}} \int_{\Omega} \gamma \nabla\left(I_{h}\left(u\left(t_{n+1}\right)\right)-u\left(t_{n+1}\right)\right) \nabla v_{h}^{n+1} d x d t-\int_{t_{n}}^{t_{n+1}} \int_{\Omega} \gamma \nabla\left(I_{h}\left(u\left(t_{n}\right)\right)-u\left(t_{n}\right)\right) \nabla a_{n+1} d x d t \\
& =\int_{t_{n}}^{t_{n+1}} \int_{\Omega} \gamma \nabla\left(g\left(t_{n+1}\right)-g\left(t_{n}\right)\right) \nabla a_{n+1} d x d t \\
& =\int_{t_{n}}^{t_{n+1}} \int_{t}^{t_{n+1}} \int_{\Omega} \gamma \nabla\left(g^{\prime}(\tau, x)\right) \nabla a_{n+1} d x d \tau d t \\
& \leq k^{2}\left|C_{\gamma}\right|\left\|g^{\prime}(\tau)\right\|_{1, \Omega}\left|a_{n+1}\right|_{1, \Omega} \\
& \leq k^{2}\left|C_{\gamma}\right| \operatorname{ch}\left\|u^{\prime}(\tau)\right\|_{2, \Omega}\left|a_{n+1}\right|_{1, \Omega} \\
& \leq k^{2} \operatorname{ch}\left|C_{\gamma}\right|\left\|u^{\prime}\right\|_{L^{\infty}\left(0, T, H^{2}(\Omega)\right)}\left|a_{n+1}\right|_{1, \Omega}
\end{aligned}
$$

Using the inequality $a b \leq \frac{1}{2 \epsilon_{3}} a^{2}+\frac{\epsilon_{3}}{2} b^{2}$,

with $a=\left|C_{\gamma}\right| \operatorname{ch} k^{\frac{3}{2}}\left\|u^{\prime}\right\|_{L^{\infty}}$ and $b=\sqrt{k}\left\|a_{n+1}\right\|_{1, \Omega}$, we get

$$
T_{4} \leq \frac{1}{2 \epsilon_{3}}\left|C_{\gamma}\right|^{2} c^{2} h^{2} k^{3}\left\|u^{\prime}\right\|_{L^{\infty}\left(0, T, H^{2}(\Omega)\right)}^{2}+\frac{\epsilon_{3}}{2} k\left|a_{n+1}\right|_{1, \Omega}^{2}
$$

Finally, the term $T_{5}$ can be bounded as

$$
\begin{aligned}
T_{5} & =\int_{t_{n}}^{t_{n+1}} \int_{\Omega} \gamma \nabla\left(u\left(t_{n}\right)\right) \nabla v_{h}^{n+1} d x d t \\
& \leq k\left|C_{\gamma}\right|\|u\|_{1, \Omega}\left|a_{n+1}\right|_{1, \Omega} \\
& \leq k\left|C_{\gamma}\right|\|u\|_{L^{\infty}\left(0, T, H^{1}(\Omega)\right)}\left|a_{n+1}\right|_{1, \Omega}
\end{aligned}
$$

Using the inequality $a b \leq \frac{1}{2 \epsilon_{4}} a^{2}+\frac{\epsilon_{4}}{2} b^{2}$,

with $a=\left|C_{\gamma}\right| k^{\frac{1}{2}}\|u\|_{L^{\infty}}$ and $b=k^{\frac{1}{2}}\left\|a_{n+1}\right\|_{1, \Omega}$, we get

$$
T_{5} \leq \frac{1}{2 \epsilon_{4}}\left|C_{\gamma}\right|^{2} k\|u\|_{L^{\infty}\left(0, T, H^{1}(\Omega)\right)}^{2}+\frac{\epsilon_{4}}{2} k\left\|a_{n+1}\right\|_{1, \Omega}^{2}
$$

Now using all the previous bounds, we obtain

$$
\int_{\Gamma} a_{n+1}^{2} d s-\int_{\Gamma} a_{n}^{2} d s+\int_{\Gamma}\left(a_{n+1}-a_{n}\right)^{2} d s+k\left|C_{\gamma}\right|\left|a_{n+1}\right|_{1, \Omega}^{2}-k\left|C_{\gamma}\right|\left|a_{n}\right|_{1, \Omega}\left|a_{n+1}\right|_{1, \Omega}
$$




$$
\begin{gathered}
\leq \frac{1}{2 \epsilon_{1}} c_{1}^{2} h^{2} k\left\|u^{\prime}(\tau)\right\|_{L^{\infty}\left(0, T, H^{2}(\Omega)\right)}^{2}+\frac{\epsilon_{1}}{2} k\left\|a_{n+1}\right\|_{0, \Gamma}^{2}+\frac{1}{2 \epsilon_{2}} k^{3}|\gamma|^{2}\left\|u^{\prime}\right\|_{L^{\infty}\left(0, T, H^{1}(\Omega)\right)}^{2}+\frac{\epsilon_{2}}{2} k\left|a_{n+1}\right|_{1, \Omega}^{2} \\
+\frac{1}{2 \epsilon_{3}}\left|C_{\gamma}\right|^{2} c^{2} h^{2} k^{3}\left\|u^{\prime}\right\|_{L^{\infty}\left(0, T, H^{2}(\Omega)\right)}^{2}+\frac{\epsilon_{3}}{2} k\left|a_{n+1}\right|_{1, \Omega}^{2}-\frac{1}{2 \epsilon_{4}}\left|C_{\gamma}\right|^{2} k\|u\|_{L^{\infty}\left(0, T, H^{1}(\Omega)\right)}^{2}-\frac{\epsilon_{4}}{2} k\left\|a_{n+1}\right\|_{1, \Omega}^{2}
\end{gathered}
$$

Choosing $\epsilon_{1}=\frac{1}{8 T}, \epsilon_{2}=\frac{\left|C_{\gamma}\right|}{2}, \epsilon_{3}=\frac{\left|C_{\gamma}\right|}{2}$ and $\epsilon_{4}=\frac{\left|C_{\gamma}\right|}{2}$, we get

$$
\begin{gathered}
\int_{\Gamma} a_{n+1}^{2} d s-\int_{\Gamma} a_{n}^{2} d s+\int_{\Gamma}\left(a_{n+1}-a_{n}\right)^{2} d s+\frac{3}{4} k\left|C_{\gamma}\right|\left|a_{n+1}\right|_{1, \Omega}^{2}-k\left|C_{\gamma}\right|\left|a_{n}\right|_{1, \Omega}\left|a_{n+1}\right|_{1, \Omega} \\
\leq c k\left(h^{2}+k^{2}\right)+\frac{k}{16 T}\left|a_{n+1}\right|_{0, \Gamma}
\end{gathered}
$$

Taking sum from $n=0,1, \ldots, m$ and replacing $A_{m}=4\left\|a_{m+1}\right\|_{0, \Gamma}^{2}, C_{m}=4 c^{\prime}\left(h^{2}+k^{2}\right)$ and $B_{m}=4 k|\gamma| \sum_{n=0}^{m}\left(\frac{3}{4}\left|a_{n+1}^{2}\right|-\left|a_{n}\right|\left|a_{n+1}\right|\right)$ with $\frac{k}{16 T} \leq \frac{1}{4}$, we get

$$
A_{m}+B_{m} \leq C_{m}+\lambda \sum_{n=0}^{m-1} A_{n}
$$

Using Gronwall's Lemma and the properties of $I_{h}$ we obtain the result.

\section{A Posteriori Error Estimate}

In this section a posteriori error estimates between the exact solution and the numerical one will be established.

Proposition 2 (Verfurth, 1996) Denote by $P_{r}(\kappa)$ the space of polynomials of degree less than $r$ on $\kappa$, we have $\forall v \in P_{r}(\kappa)$

$$
\begin{gathered}
c\|v\|_{0, \kappa} \leq\left\|v \psi_{\kappa}^{\frac{1}{2}}\right\|_{0, \kappa} \leq c^{\prime}\|v\|_{0, \kappa} \\
|v|_{1, \kappa} \leq c h_{\kappa}^{-1}\|v\|_{0, \kappa}
\end{gathered}
$$

Proposition 3 (Verfurth, 1996) Denote by $P_{r}(e)$ the space of polynomials of degree less than $r$ on e, we have $\forall v \in P_{r}(e)$,

$$
c\|v\|_{0, e} \leq\left\|v \psi_{e}^{\frac{1}{2}}\right\|_{0, e} \leq c^{\prime}\|v\|_{0, e}
$$

and for all polynomials in $P_{r}(e)$ vanishing on $\partial e$,

$$
\left\|L_{e} v\right\|_{0, \kappa}+h_{e}\left|L_{e} v\right|_{1, \kappa} \leq c h_{e}^{\frac{1}{2}}\|v\|_{0, e}
$$

For the a posteriori error estimates, consider $\forall t \in\left(t_{n-1}, t_{n}\right)$ the piecewise affine function $u_{h}(t)$ which take the values

$$
u_{h}(t)=\frac{t-t_{n-1}}{\tau_{n}}\left(u_{h}^{n}-u_{h}^{n-1}\right)+u_{h}^{n-1}
$$

The solutions of Problems (2) and (3) verify the following

$$
\begin{aligned}
T(v) & =\int_{\Omega} \gamma \nabla\left(u-u_{h}\right) \nabla v(t, x) d x+2 \int_{\Gamma} \frac{\partial\left(u-u_{h}\right)}{\partial t}(t, x) v(t, x) d x \\
& =\int_{\Omega} \gamma \nabla u \nabla v d x-\int_{\Omega} \gamma \nabla u_{h} \nabla v d x-2 \int_{\Gamma} \frac{\partial u_{h}}{\partial t}(t, x) v(t, x) d x+2 \int_{\Gamma} \frac{\partial u}{\partial t} v d x \\
& =-\int_{\Omega} \gamma \nabla u_{h} \nabla v d x-2 \int_{\Gamma} \frac{\partial u_{h}}{\partial t}(t, x) v(t, x) d x
\end{aligned}
$$

adding and subtracting $u_{h}^{n}$ and $u_{h}^{n-1}$ to the first term, then using the value of $u_{h}$ we get 


$$
\begin{aligned}
T(v) & =-\int_{\Omega} \gamma \nabla\left(u_{h}-u_{h}^{n}\right) \nabla v d x-\int_{\Omega} \gamma \nabla\left(u_{h}^{n}-u_{h}^{n-1}\right) \nabla v d x-\int_{\Omega} \nabla u_{h}^{n-1} \nabla v d x-2 \int_{\Gamma} \frac{u_{h}^{n}-u_{h}^{n-1}}{\tau_{n}}(t, x) v(t, x) d x \\
& =-\frac{t-t_{n}}{\tau_{n}} \int_{\Omega} \gamma \nabla\left(u_{h}^{n}-u_{h}^{n-1}\right) \nabla v d x-\int_{\Omega} \gamma \nabla\left(u_{h}^{n}-u_{h}^{n-1}\right) \nabla v d x-\int_{\Omega} \gamma \nabla u_{h}^{n-1} \nabla v d x-2 \int_{\Gamma} \frac{u_{h}^{n}-u_{h}^{n-1}}{\tau_{n}}(t, x) v(t, x) d x \\
& =-\frac{t-t_{n-1}}{\tau_{n}} \int_{\Omega} \gamma \nabla\left(u_{h}^{n}-u_{h}^{n-1}\right) \nabla v d x-\int_{\Omega} \gamma \nabla u_{h}^{n-1} \nabla v d x-2 \int_{\Gamma} \frac{u_{h}^{n}-u_{h}^{n-1}}{\tau_{n}}(t, x) v(t, x) d x
\end{aligned}
$$

adding and subtracting $v_{h}$ to the second and third terms, we get

$$
\begin{aligned}
T(v) & =-\frac{t-t_{n-1}}{\tau_{n}} \int_{\Omega} \gamma \nabla\left(u_{h}^{n}-u_{h}^{n-1}\right) \nabla v d x-\int_{\Omega} \gamma \nabla u_{h}^{n-1} \nabla\left(v-v_{h}\right) d x-\int_{\Omega} \gamma \nabla u_{h}^{n-1} \nabla v_{h} d x \\
& -2 \int_{\Gamma} \frac{u_{h}^{n}-u_{h}^{n-1}}{\tau_{n}}\left(v-v_{h}\right) d x-2 \int_{\Gamma} \frac{u_{h}^{n}-u_{h}^{n-1}}{\tau_{n}} v_{h} d x \\
& =-\frac{t-t_{n-1}}{\tau_{n}} \int_{\Omega} \gamma \nabla\left(u_{h}^{n}-u_{h}^{n-1}\right) \nabla v d x-\int_{\Omega} \gamma \nabla u_{h}^{n-1} \nabla\left(v-v_{h}\right) d x-\int_{\Omega} \gamma \nabla u_{h}^{n} \nabla v_{h} d x \\
& -2 \int_{\Gamma} \frac{u_{h}^{n}-u_{h}^{n-1}}{\tau_{n}} v_{h} d x
\end{aligned}
$$

adding and subtracting $v$ to the third term,

$$
\begin{aligned}
T(v) & =-\frac{t-t_{n-1}}{\tau_{n}} \int_{\Omega} \gamma \nabla\left(u_{h}^{n}-u_{h}^{n-1}\right) \nabla v d x-\int_{\Omega} \gamma \nabla u_{h}^{n-1} \nabla\left(v-v_{h}\right) d x-\int_{\Omega} \gamma \nabla u_{h}^{n} \nabla\left(v-v_{h}\right) d x \\
& -2 \int_{\Gamma} \frac{u_{h}^{n}-u_{h}^{n-1}}{\tau_{n}}\left(v-v_{h}\right) d x+\int_{\Omega} \gamma \nabla u_{h}^{n} \nabla v d x
\end{aligned}
$$

Applying Green's theorem on the second and third terms we get

$$
\begin{aligned}
T(v) & =-\frac{t-t_{n-1}}{\tau_{n}} \int_{\Omega} \gamma \nabla\left(u_{h}^{n}-u_{h}^{n-1}\right) \nabla v d x-\sum_{k \in \tau_{n} h}\left(\int_{k} \operatorname{div}\left(\gamma \nabla u_{h}^{n-1}\right)\left(v-v_{h}\right) d x-\int_{\partial \kappa}\left(\nabla u_{h}^{n-1} \cdot n\right)\left(v-v_{h}\right) d x\right) \\
& -\sum_{k \in \tau_{n} h}\left(\int_{k} \operatorname{div}\left(\gamma \nabla u_{h}^{n}\right)\left(v-v_{h}\right) d x-\int_{\partial \kappa}\left(\nabla u_{h}^{n} \cdot n\right)\left(v-v_{h}\right) d x\right)+\int_{\Omega} \gamma \nabla u_{h}^{n} \nabla d x \\
& -2 \int_{\Gamma} \frac{u_{h}^{n}-u_{h}^{n-1}}{\tau_{n}}\left(v-v_{h}\right) d x \\
= & -\frac{t-t_{n-1}}{\tau_{n}} \int_{\Omega} \gamma \nabla\left(u_{h}^{n}-u_{h}^{n-1}\right) \nabla v d x-\sum_{k \in \tau_{n} h} \int_{\partial \kappa}\left(\nabla u_{h}^{n-1} \cdot n\right)\left(v-v_{h}\right) d x \\
& -\sum_{k \in \tau_{n} h} \int_{\partial \kappa}\left(\nabla u_{h}^{n} \cdot n\right)\left(v-v_{h}\right) d x+\int_{\Omega} \gamma \nabla u_{h}^{n} \nabla v d x-2 \int_{\Gamma} \frac{u_{h}^{n}-u_{h}^{n-1}}{\tau_{n}}\left(v-v_{h}\right) d x
\end{aligned}
$$

We define, for every edge e of the mesh, the function

$$
\left(\varphi_{h, n}^{e}\right)= \begin{cases}\gamma\left[\nabla u_{h}^{n} \cdot n\right]_{e} & , e \in \epsilon_{k}, \\ \gamma \nabla\left(u_{h}^{n}\right) \cdot n+\gamma \nabla\left(u_{h}^{n-1}\right) \cdot n+2 \frac{u_{h}^{n}-u_{h}^{n-1}}{\tau_{n}} & , e \in \epsilon_{k}^{m} .\end{cases}
$$

We get the following equation

$$
\int_{\Omega} \gamma \nabla\left(u-u_{h}\right) \nabla v(t, x) d x+2 \int_{\Gamma} \frac{\partial u-u_{h}}{\partial t}(t, x) v(t, x) d x
$$




$$
=\frac{t_{n-1}-t}{\tau_{n}} \int_{\Omega} \gamma \nabla\left(u_{h}^{n}-u_{h}^{n-1}\right) \nabla v d x+\int_{\Omega} \gamma \nabla u_{h}^{n-1} \nabla v d x-\sum_{k \in \tau_{h, n}} \sum_{e \in \partial k} \int_{e} \varphi_{h, n}^{e}(x)\left(v-v_{h}\right) d x
$$

For each $k$ in $\tau_{h, n}$ we introduce the indicators

$$
\begin{gathered}
\eta_{n, k}^{\tau}=\sqrt{\frac{\tau_{n}}{3}}\left\|\nabla\left(u_{h}^{n}-u_{h}^{n-1}\right)\right\|_{0, k}+\sqrt{\tau_{n}}\left\|\nabla u_{h}^{n-1}\right\|_{0, k} \\
\left(\eta_{n, k}^{h}\right)^{2}=\sum_{e \in \partial k} h_{e}\left\|\varphi_{h, n}^{e}\right\|_{0, e}^{2}
\end{gathered}
$$

\subsection{Upper Bounds of the Error}

Theorem 4 For all $m=1, \ldots, N$, we have the following upper bound

$$
c\left\|\nabla\left(u-u_{h}\right)\right\|_{L^{2}\left(0, t_{m}, L^{2}(\Omega)\right)}^{2}+\left\|u\left(t_{m}\right)-u_{h}^{m}\right\|_{0, \Gamma}^{2} \leq c^{\prime}\left[\left(\eta_{n, k}^{\tau}\right)^{2}+\sum_{n=1}^{m} \sum_{k} \tau_{n}\left(\eta_{n, k}^{h}\right)^{2}+\left\|u_{0}-u_{h}^{0}\right\|_{0, \Gamma}^{2}\right]
$$

where $c$ is a constant.

Proof. We denote by $L(v)$ the following,

$$
L(v)=\int_{\Omega} \gamma \nabla\left(u-u_{h}\right) \nabla v(t, x) d x+2 \int_{\Gamma} \frac{\partial u-u_{h}}{\partial t} v d s
$$

and we define the function $w(t, x)$ by

$$
w(t, x)=e^{-t}\left(u-u_{h}\right)(t, x)
$$

which verifies the equation

$$
\frac{\partial w}{\partial t}+w=e^{-t} \frac{\partial\left(u-u_{h}\right)}{\partial t}
$$

Multiplying $L(v)$ by $e^{-t}$ and taking $w=v$,

$$
\begin{aligned}
e^{-t} L(v) & =\int_{\Omega} \gamma \nabla\left(e^{-t}\left(u-u_{h}\right)\right) \nabla v d x+2 \int_{\Gamma} e^{-t} \frac{\partial u-u_{h}}{\partial t} v d s \\
& =\int_{\Omega} \gamma \nabla w \nabla v d x+2 \int_{\Gamma} w v d s+2 \int_{\Gamma} \frac{\partial w}{\partial t} v d s \\
& =\int_{\Omega} \gamma|\nabla w|^{2} d x+2 \int_{\Gamma} w^{2} d s+\int_{\Gamma} \frac{\partial\left(w^{2}\right)}{\partial t} d s \\
& \geq \int_{\Omega} \gamma|\nabla w|^{2} d x+\int_{\Gamma} \frac{\partial\left(w^{2}\right)}{\partial t} d s \\
& \geq c\|\nabla w\|_{0, \Omega}^{2}+\int_{\Gamma} \frac{\partial w^{2}}{\partial t} d s
\end{aligned}
$$

Note that $e^{-t} \leq 1$, so $L(w) \leq L\left(u-u_{h}\right)$, then we have the following $c\|\nabla w\|_{0, \Omega}^{2}+\int_{\Gamma} \frac{\partial w^{2}}{\partial t} d s$

$$
\leq \int_{\Omega} \nabla\left(u-u_{h}\right) \nabla\left(u-u_{h}\right) d x+\int_{\Gamma} \frac{\partial\left(u-u_{h}\right)}{\partial t}\left(u-u_{h}\right) d s
$$

Integrating in $\left(t_{n-1}, t_{n}\right)$, we get

$$
\begin{gathered}
\int_{t_{n-1}}^{t_{n}} c\|\nabla w\|_{0, \Omega}^{2} d t+\int_{t_{n-1}}^{t_{n}} \int_{\Gamma} \frac{\partial\left(w^{2}\right)}{\partial t} d s d t \leq \int_{t_{n-1}}^{t_{n}} L\left(u-u_{h}\right) d t \\
\int_{t_{n-1}}^{t_{n}} c\|\nabla w\|_{0, \Omega}^{2} d t+\int_{\Gamma} w^{2}\left(t_{n}, s\right) d s-\int_{\Gamma} w^{2}\left(t_{n-1}, s\right) d s \leq \int_{t_{n-1}}^{t_{n}} L\left(u-u_{h}\right) d t
\end{gathered}
$$

Taking the sum from 1 to $m$, we get 


$$
\begin{gathered}
c \sum_{n=1}^{m} \int_{t_{n-1}}^{t_{n}}\left\|\nabla e^{-t}\left(u-u_{h}\right)\right\|_{0, \Omega}^{2} d t-\int_{\Gamma} e^{-2 t}\left|u-u_{h}\right|^{2}(0, s) d s+\int_{\Gamma} e^{-2 t}\left|u-u_{h}\right|^{2}\left(t_{m}, s\right) d s \\
\leq \sum_{n=1}^{m} \int_{t_{n-1}}^{t_{n}} L\left(u-u_{h}\right) d t \\
e^{-2 T}\left[c \sum_{n=1}^{m}\left(\int_{t_{n-1}}^{t_{n}}\left\|\nabla\left(u-u_{h}\right)\right\|_{0, \Omega}^{2} d t+\int_{\Gamma}\left|u-u_{h}\right|^{2}\left(t_{m}, s\right) d s\right)\right] \\
\leq \sum_{n=1}^{m} \int_{t_{n-1}}^{t_{n}} L\left(u-u_{h}\right) d t+\int_{\Gamma}\left|u-u_{h}\right|^{2}(0, s) d s
\end{gathered}
$$

so that

$$
\int_{0}^{t_{m}} c\left\|\nabla\left(u-u_{h}\right)\right\|_{0, \Omega}^{2} d t+\left\|u\left(t_{m}\right)-u_{h}^{m}\right\|_{0, \Gamma}^{2} \leq c^{\prime}\left[\sum_{n=1}^{m} \int_{t_{n-1}}^{t_{n}} L\left(u-u_{h}\right) d t+\left\|u_{0}-u_{h}^{0}\right\|_{0, \Gamma}^{2}\right]
$$

We decompose $L(v)=L_{1}(v)+L_{2}(v)$ and denote $v=u-u_{h}$.

Now we have to bound $L_{1}(v)$,

$$
\begin{aligned}
L_{1}(v) & =\frac{t_{n}-t}{\tau_{n}} \int_{\Omega} \gamma \nabla\left(u_{h}^{n}-u_{h}^{n-1}\right) \nabla v d x-\int_{\Omega} \gamma \nabla u_{h}^{n-1} \nabla v d x \\
& =\frac{t_{n}-t}{\tau_{n}} \sum_{k \in \tau_{h, n}} \int_{k} \gamma \nabla\left(u_{h}^{n}-u_{h}^{n-1}\right) \nabla v d x-\sum_{k \in \tau_{h, n}} \int_{k} \gamma \nabla u_{h}^{n-1} \nabla v d x \\
& \leq\left|\frac{t_{n}-t}{\tau_{n}}\right| \sum_{k \in \tau_{h, n}} c_{\gamma}\left\|\nabla\left(u_{h}^{n}-u_{h}^{n-1}\right)\right\|_{0, k}\|\nabla v\|_{0, k}+\sum_{k \in \tau_{h, n}} c_{\gamma}\left\|\nabla v_{h}^{n-1}\right\|_{0, k}\|\nabla v\|_{0, k}
\end{aligned}
$$

Integrating in $\left(t_{n-1}, t_{n}\right)$, then taking the sum from 1 to $m$, we get

$$
\begin{aligned}
\int_{t_{n-1}}^{t_{n}} L_{1}(v) d t & \leq \sum_{k \in \tau_{h, n}}\left[\int_{t_{n-1}}^{t_{n}}\left(\left|\frac{t_{n}-t}{\tau_{n}}\right| c\left\|\nabla\left(u_{h}^{n}-u_{h}^{n-1}\right)\right\|_{0, k}\right)^{2} d t\right]^{\frac{1}{2}}\left[\int_{t_{n-1}}^{t_{n}}\|\nabla v\|_{0, k}^{2} d t\right]^{\frac{1}{2}} \\
& +\sum_{k \in \tau_{h, n}}\left[\int_{t_{n-1}}^{t_{n}}\left(c_{\gamma}\left\|\nabla v_{h}^{n-1}\right\|_{0, k}^{2} d t\right]^{\frac{1}{2}}\left[\int_{t_{n-1}}^{t_{n}}\|\nabla v\|_{0, k}^{2} d t\right]^{\frac{1}{2}}\right. \\
& =\sum_{k \in \tau_{h, n}}\left[c_{\gamma}^{2} \frac{\tau_{n}}{3}\left\|\nabla\left(u_{h}^{n}-u_{h}^{n-1}\right)\right\|_{0, k}^{2}\right]^{\frac{1}{2}}\left[\int_{t_{n-1}}^{t_{n}}\|\nabla v\|_{0, k}^{2} d t\right]^{\frac{1}{2}} \\
& +\sum_{k \in \tau_{h, n}}\left[c_{\gamma}^{2} \tau_{n}\left\|\nabla v_{h}^{n-1}\right\|_{0, k}^{2} d t\right]^{\frac{1}{2}}\left[\int_{t_{n-1}}^{t_{n}}\|\nabla v\|_{0, k}^{2} d t\right]^{\frac{1}{2}} \\
& =\left[\int_{t_{n-1}}^{t_{n}}\|\nabla v\|_{0, k}^{2} d t\right]^{\frac{1}{2}} \sum_{k \in \tau_{h, n}} c_{\gamma}\left[\sqrt{\frac{\tau_{n}}{3}}\left\|\nabla\left(u_{h}^{n}-u_{h}^{n-1}\right)\right\|_{0, k}+\sqrt{\tau_{n}}\left\|\nabla v_{h}^{n-1}\right\|_{0, k}\right] \\
& =\left[\int_{t_{n-1}}^{t_{n}}\|\nabla v\|_{0, k}^{2} d t\right]^{\frac{1}{2}} \sum_{k \in \tau_{h, n}} c_{\gamma}\left[\eta_{n, k}^{\tau}\right] \\
& =\left[\int_{t_{n-1}}^{t_{n}}\|\nabla v\|_{0, k}^{2} d t\right]^{\frac{1}{2}} \sum_{k \in \tau_{h, n}} c_{\gamma}^{2}\left[\left(\eta_{n, k}^{\tau}\right)^{2}\right]^{\frac{1}{2}}
\end{aligned}
$$

Using the inequality $a b \leq \frac{1}{2 \epsilon_{1}} a^{2}+\frac{\epsilon_{1}}{2} b^{2}$, with $a=\left(c_{\gamma}^{2}\left(\eta_{n, k}^{\tau}\right)^{2}\right)^{\frac{1}{2}}$ and $b=\left(\int_{t_{n-1}}^{t_{n}}\|\nabla v\|_{0, k}^{2} d t\right)^{\frac{1}{2}}$,

we get

$$
\int_{t_{n-1}}^{t_{n}} L_{1}(v) d t \leq \frac{1}{2 \epsilon_{1}} c_{\gamma}^{2} \sum_{k \in \tau_{h, n}}\left(\eta_{n, k}^{\tau}\right)^{2}+\frac{\epsilon_{1}}{2}\|\nabla v\|_{L^{2}\left(t_{n-1}, t_{n}, L^{2}(\Omega)\right)}^{2}
$$


Taking sum from $n=1, . ., m$, we get

$$
\sum_{n=1}^{m} \int_{t_{n-1}}^{t_{n}} L_{1}(v) d t \leq c_{\gamma} \sum_{n=1}^{m} \sum_{k \in \tau_{h, n}}\left(\eta_{n, k}^{\tau}\right)^{2}+\frac{\epsilon_{1}}{2}\left\|\nabla\left(u-u_{h}\right)\right\|_{L^{2}\left(0, t_{m}, L^{2}(\Omega)\right)}^{2}
$$

Next, we will bound $L_{2}(v)$, using the following proposition (Clement, 1975) The clément regularization operator $R_{n, h}$ : $H^{1}(\Omega) \rightarrow X_{h}$ has the following property,

$\forall k \in \tau_{n, h}$ and $\forall v \in H^{1}(\Omega)$, we have the following

$$
\left\|v-R_{n, h} v\right\|_{0, k} \leq c h_{k}\|\nabla v\|_{0, \Delta_{k}}
$$

and

$$
\begin{gathered}
\left\|v-R_{n, h} v\right\|_{0, e} \leq c h_{e}^{\frac{1}{2}}\|\nabla v\|_{0, \Delta_{e}} \\
L_{2}(v)=-\sum_{k \in \tau_{h, n}} \sum_{e \in \partial k} \int_{e} \varphi_{h, n}^{e}(x)\left(v-v_{h}\right) d x \\
\leq \sum_{k \in \tau_{h, n}} \sum_{e \in \partial k}\left\|\varphi_{h, n}^{e}\right\|_{0, e}\left\|v(t)-v_{h}(t)\right\|_{0, e}
\end{gathered}
$$

Now we take $v_{h}(t)=R_{n, h}(v(t))$, and use the above preposition to get

$$
\begin{aligned}
L_{2}(v) & \leq \sum_{k \in \tau_{h, n}} \sum_{e \in \partial k}\left\|\varphi_{h, n}^{e}\right\|_{0, e}\left\|v(t)-R_{n, h}(v(t))\right\|_{0, e} \\
& \leq \sum_{k \in \tau_{h, n}} \sum_{e \in \partial k}\left\|\varphi_{h, n}^{e}\right\|_{0, e} c_{2} h_{e}^{\frac{1}{2}}\|\nabla v(t)\|_{0, \Delta e}
\end{aligned}
$$

Using the inequality $\sum a b \leq\left(\sum a^{2}\right)^{\frac{1}{2}}\left(\sum b^{2}\right)^{\frac{1}{2}}$, with $a=h e^{\frac{1}{2}}\left\|\varphi_{h, n}^{e}\right\|_{0, e}$ and $b=\|\nabla v(t)\|_{0, \Delta e}$, we obtain

$$
\begin{aligned}
L_{2}(v) & \leq c_{2} \sum_{k \in \tau_{h, n}}\left[\sum_{e \in \partial k} h_{e}\left\|\varphi_{h, n}^{e}\right\|_{0, e}^{2}\right]^{\frac{1}{2}}\left[\sum_{e \in \partial k}\|\nabla v(t)\|_{0, \Delta e}^{2}\right]^{\frac{1}{2}} \\
& \leq c_{2}\left[\sum_{k \in \tau_{h, n}}\left(\eta_{n, k}^{h}\right)^{2}\right]^{\frac{1}{2}}\left[\sum_{k \in \tau_{h, n}} \sum_{e \in \partial k}\|\nabla v(t)\|_{0, \Delta e}^{2}\right]^{\frac{1}{2}} \\
& \leq c_{3}\left[\sum_{k \in \tau_{h, n}}\left(\eta_{n, k}^{h}\right)^{2}\right]^{\frac{1}{2}}\|\nabla v(t)\|_{0, \Omega}
\end{aligned}
$$

Integrating in $\left(t_{n-1}, t_{n}\right)$,

$$
\begin{aligned}
\int_{t_{n-1}}^{t_{n}} L_{2}(v) d t & \leq c_{3}\left[\int_{t_{n-1}}^{t_{n}} \sum_{k \in \tau_{h, n}}\left(\eta_{n, k}^{h}\right)^{2} d t\right]^{\frac{1}{2}}\left[\int_{t_{n-1}}^{t_{n}}\|\nabla v(t)\|_{0, \Omega}^{2} d t\right]^{\frac{1}{2}} \\
& \leq c_{3}\left[\sum_{k \in \tau_{h, n}} \tau_{n}\left(\eta_{n, k}^{h}\right)^{2}\right]^{\frac{1}{2}}\left[\|\nabla v\|_{L^{2}\left(t_{n-1}, t_{n}, L^{2}(\Omega)\right)}\right]
\end{aligned}
$$

Taking the sum from $n=1, \ldots, m$, we get

$$
\sum_{n=1}^{m} \int_{t_{n-1}}^{t_{n}} L_{2}(v) d t \leq c_{3}\left[\sum_{n=1}^{m} \sum_{k \in \tau_{h, n}} \tau_{n}\left(\eta_{n, k}^{h}\right)^{2}\right]^{\frac{1}{2}}\left[\|\nabla v\|_{L^{2}\left(0, t_{m}, L^{2}(\Omega)\right)}\right]
$$


Using $a b \leq \frac{1}{2 \epsilon_{2}} a^{2}+\frac{\epsilon_{2}}{2} b^{2}$,we get

$$
\begin{aligned}
\sum_{n=1}^{m} \int_{t_{n-1}}^{t_{n}} L_{2}\left(u-u_{h}\right) d t & \leq \frac{c_{3}}{2 \epsilon_{2}} \sum_{n=1}^{m} \sum_{k \in \tau_{h, n}} \tau_{n}\left(\eta_{n, k}^{h}\right)^{2}+\frac{\epsilon_{2}}{2}\left\|\nabla\left(u-u_{h}\right)\right\|_{L^{2}\left(0, t_{m}, L^{2}(\Omega)\right)}^{2} \\
& =c_{4} \sum_{n=1}^{m} \sum_{k \in \tau_{h, n}} \tau_{n}\left(\eta_{n, k}^{h}\right)^{2}+\frac{\epsilon_{2}}{2}\left\|\nabla\left(u-u_{h}\right)\right\|_{L^{2}\left(0, t_{m}, L^{2}(\Omega)\right)}^{2}
\end{aligned}
$$

Using the above bounds, and choosing $\epsilon_{1}=\frac{c}{2}$ and $\epsilon_{2}=\frac{c}{2}$ we get

$$
\begin{gathered}
c\left\|\nabla\left(u-u_{h}\right)\right\|_{L^{2}\left(0, t_{m}, L^{2}(\Omega)\right)}^{2}+\left\|u\left(t_{m}\right)-u_{h}^{m}\right\|_{0, \Gamma}^{2} \\
\leq c^{\prime}\left[\sum_{n=1}^{m} \sum_{k \in \tau_{h, n}}\left(\eta_{n, k}^{h}\right)^{2}+\sum_{n=1}^{m} \sum_{k \in \tau_{h, n}} \tau_{n}\left(\eta_{n, k}^{h}\right)^{2}+\left\|u_{0}-u_{h}^{0}\right\|_{0, \Gamma}^{2}\right]
\end{gathered}
$$

Theorem 5 For all $m=1,2, . ., N$ we have

$$
\left\|\frac{\partial\left(u-u_{h}\right)}{\partial t}\right\|_{L^{2}\left(0, t_{m}, H^{-\frac{1}{2}}(\Gamma)\right)}^{2} \leq c^{\prime}\left[\sum_{n=1}^{m} \sum_{k \in \tau_{h, n}}\left(\left(\eta_{n, k}^{\tau}\right)^{2}+\tau_{n}\left(\eta_{n, k}^{\tau}\right)^{2}\right)+\left\|u_{0}-u_{h}^{0}\right\|_{0, \Gamma}^{2}\right]
$$

where $c^{\prime}$ is a constant.

Proof. Define the functions $r(t, x) \in H^{\frac{1}{2}}(\Gamma)$ and $w(t, x)=e^{-t}\left(u-u_{h}\right)(t, x) \in H^{1}(\Omega)$, and consider the problem

$$
\begin{cases}\operatorname{div}(\gamma \nabla w(t, x))=0 & , \text { in }(0, T) \times \Omega, \\ w(t, x)=r(t, x) & , \text { on }(0, T) \times \Gamma .\end{cases}
$$

which admits a unique solution $w(t) \in H^{1}(\Omega)$ verifying,

$$
\|\nabla w(t)\|_{0, \Omega} \leq c_{1}\|r\|_{\frac{1}{2}, \Gamma}
$$

Consider the equation

$$
\begin{gathered}
\int_{\Omega} \gamma \nabla\left(u-u_{h}\right) \nabla v(t, x) d x+2 \int_{\Gamma} \frac{\partial u-u_{h}}{\partial t} v d s \\
=\frac{t_{n}-t}{\tau_{n}} \int_{\Omega} \gamma \nabla\left(u_{h}^{n}-u_{h}^{n-1}\right) \nabla v d x-\int_{\Omega} \gamma \nabla u_{h}^{n-1} \nabla v d x-\sum_{k \in \tau_{h, n}} \sum_{e \in \partial k} \int_{e} \varphi_{h, n}^{e}(x)\left(v-v_{h}\right) d x
\end{gathered}
$$

Using the inequalities

$$
L_{1}(v) \leq\left|\frac{t_{n}-t}{\tau_{n}}\right| \sum_{k \in \tau_{h, n}} c\left\|\nabla\left(u_{h}^{n}-u_{h}^{n-1}\right)\right\|_{0, k}\|\nabla v\|_{0, k}+\sum_{k \in \tau_{h, n}} c\left\|\nabla v_{h}^{n-1}\right\|_{0, k}\|\nabla v\|_{0, k}
$$

and

we get

$$
L_{2}(v) \leq c_{3}\left[\sum_{k \in \tau_{h, n}}\left(\eta_{n, k}^{h}\right)^{2}\right]^{\frac{1}{2}}\|\nabla v(t)\|_{0, \Omega}
$$

$$
\begin{gathered}
\int_{\Omega} \gamma \nabla\left(u-u_{h}\right) \nabla v(t, x) d x+2 \int_{\Gamma} \frac{\partial\left(u-u_{h}\right)}{\partial t} v d s \\
\leq\|\nabla v(t)\|_{0, \Omega}\left[\left|\frac{t_{n}-t}{\tau_{n}}\right| \sum_{k \in \tau_{h, n}} c\left\|\nabla\left(u_{h}^{n}-u_{h}^{n-1}\right)\right\|_{0, k}+\sum_{k \in \tau_{h, n}} c\left\|\nabla u_{h}^{n-1}\right\|_{0, k}+c_{3}\left(\sum_{k \in \tau_{h, n}}\left(\eta_{n, k}^{h}\right)^{2}\right)^{\frac{1}{2}}\right]
\end{gathered}
$$

dividing by $\|v\|_{1, \Omega}$ then using Cauchy-Schwartz inequality, we get

$$
\frac{2}{\|v(t)\|_{1, \Omega}} \int_{\Gamma} \frac{\partial\left(u-u_{h}\right)}{\partial t} v(t, s) d s
$$




$$
\leq c_{\gamma}\left\|\nabla\left(u-u_{h}\right)\right\|_{0, \Omega}+c_{\gamma}\left|\frac{t_{n-1}-t}{\tau_{n}}\right|\left(\sum_{\kappa}\left\|\nabla\left(u_{h}-u_{h}^{n-1}\right)\right\|_{0, k}^{2}\right)^{\frac{1}{2}}+c\left(\sum_{\kappa}\left(\eta_{n, k}^{h}\right)^{2}\right)^{\frac{1}{2}}+\left(\sum_{\kappa} c_{\gamma}\left\|\nabla u_{h}^{n}\right\|_{0, \Omega}^{2}\right)^{\frac{1}{2}}
$$

For every $r(t) \in H^{\frac{1}{2}}(\Gamma)$, consider the harmonic lifting in $v \in H^{1}(\Omega)$ satisfying,

$$
\begin{cases}\operatorname{div}(\gamma \nabla v(t, x))=0 & , \text { in }(0, T) \times \Omega \\ v(t, x)=r(t, x) & , \text { on }(0, T) \times \Gamma\end{cases}
$$

where

$$
\|v(t)\|_{1, \Omega} \leq c_{1}\|r\|_{\frac{1}{2}, \Gamma}=c_{1}\|v\|_{\frac{1}{2}, \Gamma}
$$

SO

but

$$
\frac{1}{\|v(t)\|_{\frac{1}{2}, \Gamma}} \leq \frac{1}{\|v(t)\|_{1, \Omega}}
$$

$$
\sup _{v \in H^{\frac{1}{2}}(\Gamma)} \frac{\int_{\Gamma} \frac{\partial\left(u-u_{h}\right)}{\partial t} v d s}{\|v\|_{\frac{1}{2}, \Gamma}}=\left\|\frac{\partial\left(u-u_{h}\right)}{\partial t}\right\|_{-\frac{1}{2}, \Gamma}
$$

therefore, after integrating over $\left(t_{n-1}, t_{n}\right)$, and taking sum from $n=1, . ., m$ we get

$$
\left\|\frac{\partial\left(u-u_{h}\right)}{\partial t}\right\|_{L^{2}\left(0, t_{m}, H^{-\frac{1}{2}}(\Gamma)\right)}^{2} \leq c^{\prime}\left[\sum_{n=1}^{m} \sum_{k \in \tau_{h, n}}\left(\left(\eta_{n, k}^{\tau}\right)^{2}+\tau_{n}\left(\eta_{n, k}^{\tau}\right)^{2}\right)+\left\|u_{0}-u_{h}^{0}\right\|_{0, \Gamma}^{2}\right]
$$

Theorem 6 For all $m=1, \ldots, N$, we have the following

$$
\left\|\nabla\left(u-\pi_{\tau} u_{h}\right)\right\|_{L^{2}\left(0, t_{m}, L^{2}(\Omega)\right)}^{2} \leq c\left[\sum_{n=1}^{m} \sum_{k \in \tau_{h, n}}\left(\left(\eta_{n, k}^{\tau}\right)^{2}+\tau_{n}\left(\eta_{n, k}^{\tau}\right)^{2}\right)+\left\|u_{0}-u_{h}^{0}\right\|_{0, \Gamma}^{2}\right]
$$

where $c$ is a constant.

Proof. We have, using the previous theorem, the following bound

$$
\begin{aligned}
\left\|\nabla\left(u-\pi_{\tau} u_{h}\right)\right\|_{L^{2}} & =\left\|\nabla\left(u-u_{h}+u_{h}-\pi_{\tau} u_{h}\right)\right\|_{L^{2}} \\
& \leq\left\|\nabla\left(u-u_{h}\right)\right\|_{L^{2}}+\left\|\nabla\left(u_{h}-\pi_{\tau} u_{h}\right)\right\|_{L^{2}} \\
& \leq c^{\prime}\left[\sum_{n=1}^{m} \sum_{k \in \tau_{h, n}}\left(\left(\eta_{n, k}^{\tau}\right)^{2}+\tau_{n}\left(\eta_{n, k}^{\tau}\right)^{2}\right)+\left\|u_{0}-u_{h}^{0}\right\|_{0, \Gamma}^{2}\right]^{\frac{1}{2}}+\left\|\nabla\left(u_{h}-\pi_{\tau} u_{h}\right)\right\|_{L^{2}}
\end{aligned}
$$

Now we have to bound $\left\|\nabla\left(u_{h}-\pi_{\tau} u_{h}\right)\right\|_{L^{2}}$.

For $t \in\left(t_{n-1}, t_{n}\right)$, we have $\pi_{\tau} u_{h}(t)=u_{h}^{n}$ and $u_{h}-u_{h}^{n}=\frac{t-t_{n}}{\tau_{n}}\left(u_{h}^{n}-u_{h}^{n-1}\right)$, we have

$$
\begin{aligned}
\left\|\nabla\left(u_{h}-\pi_{\tau} u_{h}\right)\right\|_{0, \Omega}^{2} & \leq \frac{\left(t-t_{n}\right)^{2}}{\tau_{n}^{2}}\left[\sum_{k}\left\|\nabla\left(u_{h}^{n}-u_{h}^{n-1}\right)\right\|_{0, k}^{2}\right] \\
& \leq \frac{\left(t-t_{n}\right)^{2}}{\tau_{n}^{2}}\left[\sum_{k}\left\|\nabla\left(u_{h}^{n}-u_{h}^{n-1}\right)\right\|_{0, k}^{2}\right]+\tau_{n}^{2}\left\|\nabla u_{h}^{n-1}\right\|_{0, k}^{2}
\end{aligned}
$$

integrating over $\left(t_{n-1}, t_{n}\right)$, we get

$$
\begin{aligned}
\int_{t_{n-1}}^{t_{n}}\left\|\nabla\left(u_{h}-\pi_{\tau} u_{h}\right)\right\|_{0, \Omega}^{2} d t & \leq \int_{t_{n-1}}^{t_{n}} \frac{\left(t-t_{n}\right)^{2}}{\tau_{n}^{2}}\left[\sum_{k}\left\|\nabla\left(u_{h}^{n}-u_{h}^{n-1}\right)\right\|_{0, k}^{2}\right]+\tau_{n}^{2}\left\|\nabla u_{h}^{n-1}\right\|_{0, k}^{2} \\
& \leq c_{1} \sum_{k}\left(\eta_{n, k}^{\tau}\right)^{2}
\end{aligned}
$$


Finally we conclude

$$
\left\|\nabla\left(u-\pi_{\tau} u_{h}\right)\right\|_{L^{2}\left(0, t_{m}, L^{2}(\Omega)\right)} \leq c\left[\sum_{n=1}^{m} \sum_{k \in \tau_{h, n}}\left(\left(\eta_{n, k}^{\tau}\right)^{2}+\tau_{n}\left(\eta_{n, k}^{\tau}\right)^{2}\right)+\left\|u_{0}-u_{h}^{0}\right\|_{0, \Gamma}^{2}\right]^{\frac{1}{2}}
$$

\subsection{Upper Bounds of the Indicators}

Theorem 7 For all $m=1, . ., N$ we have the following estimate

$$
\left(\eta_{n, k}^{\tau}\right)^{2} \leq\left\|\nabla\left(u-\pi_{\tau} u_{h}\right)\right\|_{L^{2}\left(t_{n-1}, t_{n}, L^{2}(k)\right)}^{2}+\left\|\nabla\left(u-u_{h}\right)\right\|_{L^{2}\left(t_{n-1}, t_{n}, L^{2}(k)\right)}^{2}
$$

Proof. We have

$$
\begin{aligned}
\frac{t-t_{n}}{\tau_{n}} \nabla\left(u_{h}^{n}-u_{h}^{n-1}\right) & =\nabla\left(u_{h}-\pi_{\tau} u_{h}\right) \\
& =\nabla\left(u-u_{h}\right)+\nabla\left(u-\pi_{\tau} u_{h}\right)
\end{aligned}
$$

then

$$
\begin{aligned}
\left|\frac{t-t_{n}}{\tau_{n}} \nabla\left(u_{h}^{n}-u_{h}^{n-1}\right)\right|^{2} & =\left|\nabla\left(u-u_{h}\right)+\nabla\left(u-\pi_{\tau} u_{h}\right)\right| \\
& \leq\left|\nabla\left(u-u_{h}\right)\right|^{2}+\left|\nabla\left(u-\pi_{\tau} u_{h}\right)\right|^{2}
\end{aligned}
$$

but

$$
\left(\frac{t-t_{n}}{\tau_{n}}\right)^{2}\left|\nabla\left(u_{h}^{n}-u_{h}^{n-1}\right)\right|^{2} \leq\left(\frac{t-t_{n}}{\tau_{n}}\right)^{2}\left|\nabla\left(u_{h}^{n}-u_{h}^{n-1}\right)\right|^{2}+\left|\nabla u_{h}^{n-1}\right|^{2}
$$

integrating over $\mathrm{k}$ and on $\left(t_{n-1}, t_{n}\right)$ we get

$$
\int_{t_{n-1}}^{t_{n}} \int_{k}\left(\frac{t-t_{n}}{\tau_{n}}\right)^{2}\left|\nabla\left(u_{h}^{n}-u_{h}^{n-1}\right)\right|^{2}+\int_{t_{n-1}}^{t_{n}} \int_{k}\left|\nabla u_{h}^{n-1}\right|^{2} \leq \int_{t_{n-1}}^{t_{n}} \int_{k}\left|\nabla\left(u-u_{h}\right)\right|^{2}+\left|\nabla\left(u-\pi_{\tau} u_{h}\right)\right|^{2}
$$

then

$$
\frac{\tau_{n}}{3}\left\|\nabla\left(u_{h}^{n}-u_{h}^{n-1}\right)\right\|_{0, k}^{2}+\tau_{n}\left\|\nabla u_{h}^{n-1}\right\|_{0, k}^{2} \leq\left\|\nabla\left(u-\pi_{\tau} u_{h}\right)\right\|_{L^{2}\left(t_{n-1}, t_{n}, L^{2}(k)\right)}^{2}+\left\|\nabla\left(u-u_{h}\right)\right\|_{L^{2}\left(t_{n-1}, t_{n}, L^{2}(k)\right)}^{2}
$$

REMARK: The numerical simulation will be done in a forthcoming paper.

\section{References}

Cherif, M. A., El Arwadi, T., Emmamirad, H., \& Sac- Epee J. M. (2014). Dirichlet-to-Neumann semigroup acts as a magnifying glass. Semigroup Forum, 88(3), 753-767.

Clement, P. (1975). Approximation by finite element functions using local regularization. R.A.I.R.O. Anal. Numer., 9 , 77-84.

EL Arwadi, T., DIB, S., \& SAYAH, T. (2015). A priori and a posteriori analysis for a linear elliptic problem with dynamic boundary condition. Appl. Math. Inf. Sci., 9(6), 3305317.

Emmamirad, H., \& Shariftabar, M. (2013). On Explicit representation and Approximation of Dirichlet-to-Neumann Semigroup. Semigroup Forum, 86(1), 192-201.

Hecht, F. (2012). New development in Freefem++. Journal of Numerical Mathematics, 20, 251-266.

Lax, P. D. (2002). Functional Analysis, Wiley Inter-science, New-York.

Mishra, V. N. (2007). Some Problems on Approximations of Functions in Banach Spaces, Ph.D. Thesis, Indian Institute of Technology, Roorkee - 247 667, Uttarakhand, India.

Mishra, V. N., \& Mishra, L. N. (2012). Trigonometric Approximation of Signals (Functions) in Lpp $p_{1}$ norm. International Journal of Contemporary Mathematical Sciences, 7(19), 909 C 918. 
Mishra, V. N., Khan, H. H., Khatri, K., \& Mishra, L. N. (2013). Degree of conjugate of signals (functions) belonging to the generalized weighted Lipschitz class by $(\mathrm{C}, 1)(\mathrm{E}, \mathrm{q})$ means if conjugate trigonometric Fourier series. Bulletin of Mathematical Analysis and Applications, 5(4), 40-53.

Ostrowski, A. (1940). Recherches sur la méthode de Graeffe et les zéros des polynômes et des séries des Laurent. Acta Math., $72,9957$.

Verfurth, R. (1996). A posteriori error estimates and Adaptive Mesh-Refinement Techniques, Wiley and Teubner mathematics.

Vrabie, I. I. (2003). $C_{0}$-Semigroups and applications, North- Holland, Amsterdam.

\section{Copyrights}

Copyright for this article is retained by the author(s), with first publication rights granted to the journal.

This is an open-access article distributed under the terms and conditions of the Creative Commons Attribution license (http://creativecommons.org/licenses/by/3.0/). 